\title{
Hyperbolic Equations in Uniform Spaces
}

by

\author{
J. W. CHOLEWA and Tomasz DLOTKO
}

Presented by Andrzej LASOTA

Summary. The paper is devoted to the Cauchy problem for a semilinear damped wave equation in the whole of $\mathbb{R}^{n}$. Under suitable assumptions a bounded dissipative semigroup of global solutions is constructed in a locally uniform space $\dot{H}_{\mathrm{lu}}^{1}\left(\mathbb{R}^{n}\right) \times \dot{L}_{\mathrm{lu}}^{2}\left(\mathbb{R}^{n}\right)$. Asymptotic compactness of this semigroup and the existence of a global attractor are then shown.

1. Introduction. The uniform spaces, which can be traced back to $[\mathrm{K}]$, have already been used by many authors in the study of both parabolic and hyperbolic problems (see e.g. $[\mathrm{M}],[\mathrm{M}-\mathrm{S}],[\mathrm{F}]$ ). In this paper we develop the ideas of $[\mathrm{F}]$ for the Cauchy problem for a semilinear wave equation

$$
\left\{\begin{array}{l}
u_{t t}+\eta u_{t}-\Delta u=f(u)+g(x), \quad t>0, x \in \mathbb{R}^{n}, \\
u(0, x)=u_{0}(x), \quad u_{t}(0, x)=v_{0}(x), \quad x \in \mathbb{R}^{n} .
\end{array}\right.
$$

By a functional analytic approach we construct a semigroup $\{T(t)\}$ of global solutions corresponding to (1) in locally uniform spaces and prove the existence of an absorbing set. We also show the validity of an appropriate asymptotic compactness condition and prove the existence of a global attractor for $\{T(t)\}$. Mention should be made that in contrast to [F] we do not impose any monotonicity condition on $f$ (see Remark 4.3). Our assumptions concerning $f$ are borrowed from $[\mathrm{T}]$, where they were introduced for the damped wave equation in a bounded domain.

The results of the paper can be summarized as follows. After a finite time $t_{B}$ the image $T(t) B$ of any bounded set $B \subset \dot{H}_{\mathrm{lu}}^{2}\left(\mathbb{R}^{n}\right) \times \dot{H}_{\mathrm{lu}}^{1}\left(\mathbb{R}^{n}\right)$ will enter an absorbing set $\mathcal{B}_{0}$, bounded in $\dot{H}_{\mathrm{lu}}^{2}\left(\mathbb{R}^{n}\right) \times \dot{H}_{\text {lu }}^{1}\left(\mathbb{R}^{n}\right)$. With further increase of

2000 Mathematics Subject Classification: 35L15, 35B40, 35B41.

Key words and phrases: wave equation, uniform spaces, dissipativeness, global attractor.

Research of T. Dlotko partially supported by grant \#2001/03735-4 FAPESP, Brazil. 
time, $T(t) B$ will be attracted — in a weaker topology of $H_{\varrho}^{1}\left(\mathbb{R}^{n}\right) \times L_{\varrho}^{2}\left(\mathbb{R}^{n}\right)$ 一by a global attractor $\mathcal{A}$, which is bounded in $\dot{H}_{\mathrm{lu}}^{1}\left(\mathbb{R}^{n}\right) \times \dot{L}_{\mathrm{lu}}^{2}\left(\mathbb{R}^{n}\right)$ and compact in $H_{\varrho}^{1}\left(\mathbb{R}^{n}\right) \times L_{\varrho}^{2}\left(\mathbb{R}^{n}\right)$. In addition $\mathcal{A}$ is invariant with respect to the group of translations in $\mathbb{R}^{n}$.

\section{Well posedness of the wave equation in uniform spaces}

2.1. Wave operator and linear wave equation in uniform spaces. We begin with the Cauchy problem for the homogeneous linear equation

$$
\left\{\begin{array}{l}
u_{t t}+\eta u_{t}-\Delta u=0, \quad t>0, \quad x \in \mathbb{R}^{n}, \\
u(0, x)=u_{0}(x), \quad u_{t}(0, x)=v_{0}(x), \quad x \in \mathbb{R}^{n},
\end{array}\right.
$$

where $\eta \in \mathbb{R}$. We will consider (2) in the form of an initial value problem for an abstract ordinary differential equation

$$
\frac{d}{d t}\left[\begin{array}{l}
u \\
v
\end{array}\right]=A_{\eta}\left[\begin{array}{l}
u \\
v
\end{array}\right], \quad\left[\begin{array}{l}
u \\
v
\end{array}\right]_{t=0}=\left[\begin{array}{l}
u_{0} \\
v_{0}
\end{array}\right],
$$

in the Banach space $X=\dot{H}_{\mathrm{lu}}^{1}\left(\mathbb{R}^{n}\right) \times \dot{L}_{\mathrm{lu}}^{2}\left(\mathbb{R}^{n}\right)$ with $A_{\eta}$ defined in formula (5) below.

For a strictly positive continuous weight function $\varrho: \mathbb{R}^{n} \rightarrow(0, \infty)$ and $1 \leq p<\infty$ we set

$$
L_{\varrho}^{p}\left(\mathbb{R}^{n}\right)=\left\{\phi \in L_{\mathrm{loc}}^{p}\left(\mathbb{R}^{n}\right) ;\|\phi\|_{L_{\varrho}^{p}\left(\mathbb{R}^{n}\right)}=\left(\int_{\mathbb{R}^{n}}|\phi(x)|^{p} \varrho(x) d x\right)^{1 / p}<\infty\right\} .
$$

We consider the translated weight functions

$$
\tau_{y} \varrho(x)=\varrho(x-y), \quad y \in \mathbb{R}^{n},
$$

and the locally uniform spaces

$$
\begin{aligned}
& L_{\mathrm{lu}}^{p}\left(\mathbb{R}^{n}\right)=\left\{\phi \in L_{\mathrm{loc}}^{p}\left(\mathbb{R}^{n}\right) ;\|\phi\|_{L_{\mathrm{lu}}^{p}\left(\mathbb{R}^{n}\right)}=\sup _{y \in \mathbb{R}^{n}}\|\phi\|_{L_{\tau y \varrho}^{p}\left(\mathbb{R}^{n}\right)}<\infty\right\}, \\
& \dot{L}_{\mathrm{lu}}^{p}\left(\mathbb{R}^{n}\right)=\left\{\phi \in L_{\mathrm{lu}}^{p}\left(\mathbb{R}^{n}\right) ;\left\|\tau_{y} \phi-\phi\right\|_{L_{\mathrm{lu}}^{p}\left(\mathbb{R}^{n}\right)} \rightarrow 0 \text { as }|y| \rightarrow 0\right\},
\end{aligned}
$$

where $\dot{L}_{\mathrm{lu}}^{p}\left(\mathbb{R}^{n}\right)$ is the closed subspace of $L_{\mathrm{lu}}^{p}\left(\mathbb{R}^{n}\right)$ consisting of all its elements that are translation continuous.

The locally uniform Sobolev spaces $W_{\mathrm{lu}}^{k, p}\left(\mathbb{R}^{n}\right)$ and $\dot{W}_{\mathrm{lu}}^{k, p}\left(\mathbb{R}^{n}\right)$ are defined as in $[\mathrm{A}-\mathrm{C}-\mathrm{D}-\mathrm{RB}]$ and $[\mathrm{M}-\mathrm{S}]$. We recall that continuous Sobolev embeddings are valid for such spaces:

$$
W_{\mathrm{lu}}^{m, p}\left(\mathbb{R}^{n}\right) \subset W_{\mathrm{lu}}^{j, q}\left(\mathbb{R}^{n}\right) \quad \text { if } j-\frac{n}{q} \leq m-\frac{n}{p}, 1<p \leq q<\infty .
$$

Also compact embeddings

$$
W_{\mathrm{lu}}^{m, p}\left(\mathbb{R}^{n}\right) \subset W_{\varrho}^{j, q}\left(\mathbb{R}^{n}\right)
$$

hold whenever $j-n / q<m-n / p, 1<p \leq q<\infty$. 
We remark that if we take any positive integrable weight function $\varrho \in$ $C^{2}\left(\mathbb{R}^{n}\right)$ such that

$$
\begin{aligned}
& \left|\frac{\partial \varrho}{\partial x_{j}}(x)\right| \leq \varrho_{0} \varrho(x), \quad x \in \mathbb{R}^{n}, j=1, \ldots, n, \\
& \left|\frac{\partial^{2} \varrho}{\partial x_{j} \partial x_{k}}(x)\right| \leq \varrho_{1} \varrho(x), \quad x \in \mathbb{R}^{n}, j, k=1, \ldots, n,
\end{aligned}
$$

$\varrho_{0}, \varrho_{1}>0$, then the locally uniform spaces $\dot{H}_{\mathrm{lu}}^{2}\left(\mathbb{R}^{n}\right), \dot{H}_{\mathrm{lu}}^{1}\left(\mathbb{R}^{n}\right), \dot{L}_{\mathrm{lu}}^{2}\left(\mathbb{R}^{n}\right)$ coincide, respectively, with the uniform spaces $\dot{H}_{U}^{2}\left(\mathbb{R}^{n}\right), \dot{H}_{U}^{1}\left(\mathbb{R}^{n}\right), \dot{L}_{U}^{2}\left(\mathbb{R}^{n}\right)$ defined from

$$
L_{U}^{2}\left(\mathbb{R}^{n}\right)=\left\{\phi \in L_{\mathrm{loc}}^{2}\left(\mathbb{R}^{n}\right) ;\|\phi\|_{L_{U}^{2}\left(\mathbb{R}^{n}\right)}=\sup _{y \in \mathbb{R}^{n}}\left(\int_{\{|x-y|<1\}}|\phi(x)|^{2} d x\right)^{1 / 2}<\infty\right\}
$$

(see $[\mathrm{A}-\mathrm{C}-\mathrm{D}-\mathrm{RB}])$. Also, choosing the particular weight function $\varrho(x)=$ $\left(1+\delta|x|^{2}\right)^{-\nu}, \delta>0, \nu>n / 2$, one can achieve the estimate

$$
|\nabla \varrho| \leq c \sqrt{\delta} \varrho
$$

for any small $\delta>0$.

Our first concern will be to show that

THEOREM 2.1. The wave operator

$$
A_{\eta}=\left[\begin{array}{cc}
0 & I \\
\Delta & -\eta I
\end{array}\right], \quad \text { where } \eta \in \mathbb{R}
$$

with the domain $D\left(A_{\eta}\right)=\dot{H}_{\mathrm{lu}}^{2}\left(\mathbb{R}^{n}\right) \times \dot{H}_{\mathrm{lu}}^{1}\left(\mathbb{R}^{n}\right)$, generates a $C^{0}$ semigroup $\{\mathcal{T}(t)\}$ of linear operators in $X$. Furthermore, the linear problem (3) with initial data in $\dot{H}_{\mathrm{lu}}^{2}\left(\mathbb{R}^{n}\right) \times \dot{H}_{\mathrm{lu}}^{1}\left(\mathbb{R}^{n}\right)$ has a unique solution

$$
\left[\begin{array}{l}
u(t) \\
v(t)
\end{array}\right]=\mathcal{T}(t)\left[\begin{array}{l}
u_{0} \\
v_{0}
\end{array}\right] \quad \text { for } t \geq 0
$$

Proof. The proof proceeds in three steps.

STEP 1. Take $Y=H^{1}\left(\mathbb{R}^{n}\right) \times L^{2}\left(\mathbb{R}^{n}\right)$ and an operator $\Lambda: D(\Lambda) \subset Y \rightarrow Y$, where

$$
\Lambda=A_{0}+C_{y}
$$

with

$$
A_{0}=\left[\begin{array}{cc}
0 & I \\
\Delta & 0
\end{array}\right], \quad C_{y}=\left[\begin{array}{cc}
0 & 0 \\
-\frac{1}{2} \frac{\Delta \tau_{y} \varrho}{\tau_{y} \varrho}-\frac{\nabla \tau_{y} \varrho}{\tau_{y} \varrho} \nabla+\frac{3}{4} \frac{\left|\nabla \tau_{y} \varrho\right|^{2}}{\tau_{y} \varrho^{2}} & -\eta I
\end{array}\right] .
$$

It is well known that $A_{0}$ with the domain $H^{2}\left(\mathbb{R}^{n}\right) \times H^{1}\left(\mathbb{R}^{n}\right)$ generates a $C_{0}$ group $\left\{\mathcal{T}_{0}(t)\right\}$ on $Y$ satisfying $\left\|\mathcal{T}_{0}(t)\right\|_{L(Y, Y)} \leq e^{|t|}$ (see $[\mathrm{P}, \S 7.4]$ ). Since $C_{y}: Y \rightarrow Y$ is a bounded linear perturbation of $A_{0}$, the operator $\Lambda$ with the domain $H^{2}\left(\mathbb{R}^{n}\right) \times H^{1}\left(\mathbb{R}^{n}\right)$ is the infinitesimal generator of a $C^{0}$ semigroup 
$\{S(t)\}$ on $Y$ satisfying $\|S(t)\|_{L(Y, Y)} \leq e^{\left(1+\left\|C_{y}\right\|_{L(Y, Y)}\right) t}$ (see $\left.[\mathrm{P}, \S 3.1]\right)$. Note that the norm $\left\|C_{y}\right\|_{L(Y, Y)}$ is bounded by a constant $C$ which depends only on the quantities $\varrho_{0}, \varrho_{1}$ and $\eta$, but is independent of $y \in \mathbb{R}^{n}$.

STEP 2. Take $X_{\tau_{y} \varrho}=H_{\tau_{y} \varrho}^{1}\left(\mathbb{R}^{n}\right) \times L_{\tau_{y} \varrho}^{2}\left(\mathbb{R}^{n}\right)$, and consider an isomorphism $\Phi: X_{\tau_{y} \varrho} \rightarrow Y$,

$$
\Phi=\left[\begin{array}{cc}
\left(\tau_{y} \varrho\right)^{1 / 2} & 0 \\
0 & \left(\tau_{y} \varrho\right)^{1 / 2}
\end{array}\right] .
$$

Since $A_{\eta}$ in $X_{\tau_{y} \varrho}$ coincides with $\Phi^{-1} \Lambda \Phi: \Phi^{-1}(D(\Lambda)) \subset X_{\tau_{y} \varrho} \rightarrow X_{\tau_{y} \varrho}$, where $\Phi^{-1}(D(\Lambda))=H_{\tau_{y} \varrho}^{2}\left(\mathbb{R}^{n}\right) \times H_{\tau_{y} \varrho}^{1}\left(\mathbb{R}^{n}\right)$, the resolvent set of $A_{\eta}$ in $X_{\tau_{y} \varrho}$ is the same as the resolvent set of $\Lambda$ in $Y$. Thus $\varrho\left(A_{\eta}\right)$ contains the ray $(1+C, \infty) \subset \mathbb{R}$ and

$$
\begin{aligned}
\left\|R\left(\lambda, A_{\eta}\right)^{k}\right\|_{L\left(X_{\tau_{y} \varrho}, X_{\tau_{y} \varrho}\right)} & =\left\|\Phi^{-1} R(\lambda, \Lambda)^{k} \Phi\right\|_{L\left(X_{\tau_{y} \varrho}, X_{\tau_{y} \varrho}\right)} \\
& \leq\left\|\Phi^{-1}\right\|_{L\left(Y, X_{\tau_{y} \varrho}\right)}\left\|R(\lambda, \Lambda)^{k}\right\|_{L(Y, Y)}\|\Phi\|_{L\left(X_{\tau_{y} \varrho}, Y\right)} \\
& \leq \frac{M}{(\lambda-1-C)^{k}} \quad \text { for } \lambda>1+C, k=1,2, \ldots
\end{aligned}
$$

Here $M$, which bounds $\|\Phi\|_{L\left(X_{\tau_{y} \varrho}, Y\right)}\left\|\Phi^{-1}\right\|_{L\left(Y, X_{\tau_{y} \varrho}\right)}$, depends on $\varrho_{0}$ and $\varrho_{1}$ but not on $y \in \mathbb{R}^{n}$. As a consequence, $A_{\eta}$ generates a $C^{0}$ semigroup $\{\mathcal{T}(t)\}$ on $X_{\tau_{y} \varrho}$ such that $\mathcal{T}(t)=\Phi^{-1} S(t) \Phi$.

STEP 3. Note that the operator $A_{\eta}: \dot{H}_{\mathrm{lu}}^{2}\left(\mathbb{R}^{n}\right) \times \dot{H}_{\mathrm{lu}}^{1}\left(\mathbb{R}^{n}\right) \subset \dot{H}_{\mathrm{lu}}^{1}\left(\mathbb{R}^{n}\right) \times$ $\dot{L}_{\text {lu }}^{2}\left(\mathbb{R}^{n}\right) \rightarrow \dot{H}_{\mathrm{lu}}^{1}\left(\mathbb{R}^{n}\right) \times \dot{L}_{\text {lu }}^{2}\left(\mathbb{R}^{n}\right)$ is closed and densely defined because $\Delta$ : $\dot{H}^{2}\left(\mathbb{R}^{n}\right) \subset \dot{L}^{2}\left(\mathbb{R}^{n}\right) \rightarrow \dot{L}^{2}\left(\mathbb{R}^{n}\right)$ has these properties (see [A-C-D-RB]). Furthermore, since the inverse operator $\left(\lambda I-A_{\eta}\right)^{-1}$ may be written formally in matrix form as

$$
\left(\lambda I-A_{\eta}\right)^{-1}=\left[\begin{array}{cc}
(\lambda+\eta)[\lambda(\lambda+\eta) I-\Delta]^{-1} & {[\lambda(\lambda+\eta) I-\Delta]^{-1}} \\
-I+\lambda(\lambda+\eta)[\lambda(\lambda+\eta) I-\Delta]^{-1} & \lambda[\lambda(\lambda+\eta) I-\Delta]^{-1}
\end{array}\right],
$$

from the properties of the Laplacian in locally uniform spaces (see [A-C-D$\mathrm{RB}])$ it is evident that $\left(\lambda I-A_{\eta}\right)^{-1}$ takes $\dot{H}_{\mathrm{lu}}^{1}\left(\mathbb{R}^{n}\right) \times \dot{L}_{\mathrm{lu}}^{2}\left(\mathbb{R}^{n}\right)$ into $\dot{H}_{\mathrm{lu}}^{2}\left(\mathbb{R}^{n}\right) \times$ $\dot{H}_{\text {lu }}^{1}\left(\mathbb{R}^{n}\right)$ for all sufficiently large $\lambda>0$. Using finally the fact that $M$ in (6) does not depend on $y \in \mathbb{R}^{n}$ we conclude that

$$
\left\|R\left(\lambda, A_{\eta}\right)^{k}\left[\begin{array}{c}
\phi \\
\psi
\end{array}\right]\right\|_{\dot{H}_{\mathrm{lu}}^{1}\left(\mathbb{R}^{n}\right) \times \dot{L}_{\mathrm{lu}}^{2}\left(\mathbb{R}^{n}\right)} \leq \frac{M(n+2)}{(\lambda-C)^{k}}\left\|\left[\begin{array}{c}
\phi \\
\psi
\end{array}\right]\right\|_{\dot{H}_{\mathrm{lu}}^{1}\left(\mathbb{R}^{n}\right) \times \dot{L}_{\mathrm{lu}}^{2}\left(\mathbb{R}^{n}\right)}
$$

for all sufficiently large $\lambda$ and all $k=1,2, \ldots$, which completes the proof.

2.2. Mild and classical solutions of the semilinear wave equation. In the Banach space $X=\dot{H}_{\mathrm{lu}}^{1}\left(\mathbb{R}^{n}\right) \times \dot{L}_{\mathrm{lu}}^{2}\left(\mathbb{R}^{n}\right)$ consider the semilinear Cauchy 
problem

$$
\left\{\begin{array}{l}
u_{t t}+\eta u_{t}-\Delta u=f(u)+g(x), \quad t>0, x \in \mathbb{R}^{n}, \\
u(0, x)=u_{0}(x), \quad u_{t}(0, x)=v_{0}(x), \quad x \in \mathbb{R}^{n},
\end{array}\right.
$$

where $\eta \in \mathbb{R}, g \in \dot{L}_{\mathrm{lu}}^{2}\left(\mathbb{R}^{n}\right)$ and $f: \mathbb{R} \rightarrow \mathbb{R}$ satisfies

$$
\left|f\left(s_{1}\right)-f\left(s_{2}\right)\right| \leq c\left|s_{1}-s_{2}\right|\left(1+\left|s_{1}\right|^{q-1}+\left|s_{2}\right|^{q-1}\right), \quad s_{1}, s_{2} \in \mathbb{R},
$$

for some $q \in[1, n /(n-2)]$ if $n \geq 3$ or any finite $q \geq 1$ if $n=1,2$.

Rewrite (8) as

$$
\frac{d}{d t}\left[\begin{array}{l}
u \\
v
\end{array}\right]=A_{\eta}\left[\begin{array}{l}
u \\
v
\end{array}\right]+\mathcal{F}\left(\left[\begin{array}{l}
u \\
v
\end{array}\right]\right), \quad\left[\begin{array}{l}
u \\
v
\end{array}\right]_{t=0}=\left[\begin{array}{l}
u_{0} \\
v_{0}
\end{array}\right] \in X .
$$

From (9), Sobolev embeddings and the Young inequality it is evident that the map

$$
\mathcal{F}\left(\left[\begin{array}{l}
u \\
v
\end{array}\right]\right)=\left[\begin{array}{c}
0 \\
f(u)+g
\end{array}\right]
$$

from $X$ into $X$ is Lipschitz continuous on bounded sets. Thus, application of a standard perturbation result (see $[\mathrm{P}, \S 6.1]$ ) leads to the following observation concerning mild solutions to (10).

Proposition 2.2. If $f$ satisfies (9) and $g \in \dot{L}_{\mathrm{lu}}^{2}\left(\mathbb{R}^{n}\right)$, then the problem (10) is locally uniquely solvable in $X=\dot{H}_{\mathrm{lu}}^{1}\left(\mathbb{R}^{n}\right) \times \dot{L}_{\mathrm{lu}}^{2}\left(\mathbb{R}^{n}\right)$, i.e. there exists $\tau>0$ and a unique $X$-valued function continuous on $[0, \tau]$ such that the equation

$$
\left[\begin{array}{l}
u(t) \\
v(t)
\end{array}\right]=\mathcal{T}(t)\left[\begin{array}{l}
u_{0} \\
v_{0}
\end{array}\right]+\int_{0}^{t} \mathcal{T}(t-s) \mathcal{F}\left(\left[\begin{array}{l}
u(s) \\
v(s)
\end{array}\right]\right) d s
$$

is satisfied for $t \in[0, \tau]$.

Furthermore, the mild solution to (10) is defined for all $t \geq 0$ unless its $\dot{H}_{\mathrm{lu}}^{1}\left(\mathbb{R}^{n}\right) \times \dot{L}_{\mathrm{lu}}^{2}\left(\mathbb{R}^{n}\right)$-norm blows up in a finite time.

It was shown in $[\mathrm{P}, \S 6.1]$ that if the nonlinear term $\mathcal{F}$ is a $C^{1}$ map from $X$ to $X$, then the mild solutions corresponding to initial data from $D\left(A_{\eta}\right)=\dot{H}_{\mathrm{lu}}^{2}\left(\mathbb{R}^{n}\right) \times \dot{H}_{\mathrm{lu}}^{1}\left(\mathbb{R}^{n}\right)$ are in fact classical solutions to (10) such that

$$
\begin{aligned}
& {\left[\begin{array}{l}
u\left(\cdot, u_{0}, v_{0}\right) \\
v\left(\cdot, u_{0}, v_{0}\right)
\end{array}\right] \in C\left([0, \tau], \dot{H}_{\mathrm{lu}}^{1}\left(\mathbb{R}^{n}\right) \times \dot{L}_{\mathrm{lu}}^{2}\left(\mathbb{R}^{n}\right)\right)} \\
& \cap C^{1}\left((0, \tau), \dot{H}_{\mathrm{lu}}^{1}\left(\mathbb{R}^{n}\right) \times \dot{L}_{\mathrm{lu}}^{2}\left(\mathbb{R}^{n}\right)\right), \\
& {\left[\begin{array}{l}
u\left(t, u_{0}, v_{0}\right) \\
v\left(t, u_{0}, v_{0}\right)
\end{array}\right] \in \dot{H}_{\mathrm{lu}}^{2}\left(\mathbb{R}^{n}\right) \times \dot{H}_{\mathrm{lu}}^{1}\left(\mathbb{R}^{n}\right), \quad t \in[0, \tau] .}
\end{aligned}
$$

We remark that the Fréchet derivative of $\mathcal{F}$ exists and

$$
\mathcal{F}^{\prime}\left(\left[\begin{array}{l}
u \\
v
\end{array}\right]\right)\left[\begin{array}{l}
h_{1} \\
h_{2}
\end{array}\right]=\left[\begin{array}{c}
0 \\
f^{\prime}(u) h_{1}
\end{array}\right], \quad\left[\begin{array}{l}
u \\
v
\end{array}\right],\left[\begin{array}{l}
h_{1} \\
h_{2}
\end{array}\right] \in X,
$$


provided that

$$
f \in C^{1}(\mathbb{R}), \quad\left|f^{\prime}(s)\right| \leq c\left|s_{1}-s_{2}\right|\left(1+\left|s_{1}\right|^{p-1}+\left|s_{2}\right|^{p-1}\right), \quad s_{1}, s_{2} \in \mathbb{R},
$$

where

$$
p \in[1,2] \text { if } n=3, \quad p \geq 1 \text { arbitrarily large if } n=1,2 .
$$

Proposition 2.3. If $n \leq 3$, (13) and (14) hold, $u_{0} \in \dot{H}_{\mathrm{lu}}^{2}\left(\mathbb{R}^{n}\right), v_{0} \in$ $\dot{H}_{\mathrm{lu}}^{1}\left(\mathbb{R}^{n}\right)$ and $g \in \dot{L}_{\mathrm{lu}}^{2}\left(\mathbb{R}^{n}\right)$, then the mild solution resulting from Proposition 2.2 is a classical solution.

Recall also that if $\left[\begin{array}{l}u \\ v\end{array}\right]$ is a classical solution to (9) then, as shown in the proof of $\left[\mathrm{P}\right.$, Theorem 1.5], the function $[0, \tau) \ni t \mapsto\left[\begin{array}{c}u_{t} \\ v_{t}\end{array}\right]=:\left[\begin{array}{c}v \\ w\end{array}\right] \in X$ satisfies the integral equation

$$
\begin{aligned}
& {\left[\begin{array}{c}
v \\
w
\end{array}\right]=\mathcal{T}(t)\left[\begin{array}{c}
v(0) \\
w(0)
\end{array}\right]+\int_{0}^{t} \mathcal{T}(t-s)\left[\begin{array}{c}
0 \\
f^{\prime}(u(s)) v(s)
\end{array}\right] d s} \\
& {\left[\begin{array}{c}
v(0) \\
w(0)
\end{array}\right]=\left[\begin{array}{c}
v_{0} \\
\Delta u_{0}-\eta v_{0}+f\left(u_{0}\right)+g
\end{array}\right] .}
\end{aligned}
$$

Thus, under the assumptions of Proposition 2.3, any solution $\left[\begin{array}{l}u \\ v\end{array}\right]$ corresponding to initial data from $D\left(A_{\eta}\right)$ is time differentiable (strongly in $X$ ) and its derivative is a mild solution of the Cauchy problem obtained by formal differentiation of (10).

REMARK 2.4. Clearly, it is possible to consider other spaces of initial data, like e.g. $\mathcal{X}^{s+1 / 2} \times \mathcal{X}^{s}, s \in \mathbb{R}$, with $\mathcal{X}^{s}$ from a scale of fractional power spaces corresponding to $-\Delta+I: \dot{H}_{\mathrm{lu}}^{2}\left(\mathbb{R}^{n}\right) \subset \dot{L}_{\mathrm{lu}}^{2}\left(\mathbb{R}^{n}\right) \rightarrow \dot{L}_{\mathrm{lu}}^{2}\left(\mathbb{R}^{n}\right)$.

\section{Global solvability and dissipativeness of the damped wave} equation. Due to Proposition 2.2 a uniform in time estimate of $\|(u, v)\|_{X}$ is needed to justify global solvability of (8). Below, such an estimate is derived under the assumptions used in [T, p. 207] for a damped wave equation in a bounded domain.

3.1. A priori estimate in $X$. Consider $\eta>0$ in (8) and assume the following dissipativeness conditions:

$$
\begin{aligned}
& \exists_{k \geq 1, \mu_{1}>0} \forall_{\mu \in\left(0, \mu_{1}\right]} \exists_{C_{\mu} \in \mathbb{R}} \forall_{s \in \mathbb{R}} \quad s f(s)-k F(s) \leq-\mu s^{2}+C_{\mu}, \\
& \exists_{\nu_{1}>0} \forall_{\nu \in\left(0, \nu_{1}\right]} \exists_{C_{\nu}^{\prime} \in \mathbb{R}} \forall_{s \in \mathbb{R}} \quad F(s) \leq-\nu s^{2}+C_{\nu}^{\prime},
\end{aligned}
$$

where $F(s)=\int_{0}^{s} f(z) d z\left(^{1}\right)$.

$\left({ }^{1}\right)$ The function $f(s)=s-s|s|^{q-1}, q>1$, is an example of a nonlinearity satisfying the above conditions. 
Fix a number $\varepsilon$ satisfying

$$
0<\varepsilon<\frac{\eta-\varepsilon}{4}, \quad 2 \varepsilon(\eta-\varepsilon) \leq \mu_{1},
$$

decompose $\eta=\varepsilon+(\eta-\varepsilon)$ and multiply the equation $(8)$ by $\left(u_{t}+\varepsilon u\right) \tau_{y} \varrho$ to get

$$
\begin{aligned}
\int_{\mathbb{R}^{n}}\left(u_{t t}+\varepsilon u_{t}\right)\left(u_{t}+\varepsilon u\right) \tau_{y} \varrho d x+( & \eta-\varepsilon) \int_{\mathbb{R}^{n}} u_{t}\left(u_{t}+\varepsilon u\right) \tau_{y} \varrho d x \\
& =\int_{\mathbb{R}^{n}}(\Delta u+f(u)+g)\left(u_{t}+\varepsilon u\right) \tau_{y} \varrho d x .
\end{aligned}
$$

Obvious calculations show that

$$
\begin{aligned}
& \frac{d}{d t} \mathcal{L}\left(u, u_{t}\right) \\
& :=\frac{d}{d t} \int_{\mathbb{R}^{n}}\left[\frac{1}{2}\left(u_{t}+\varepsilon u\right)^{2}+\frac{1}{2} \varepsilon(\eta-\varepsilon) u^{2}+\frac{1}{2}|\nabla u|^{2}-F(u)\right] \tau_{y} \varrho d x \\
& =-(\eta-\varepsilon) \int_{\mathbb{R}^{n}} u_{t}^{2} \tau_{y} \varrho d x+\varepsilon \int_{\mathbb{R}^{n}} u f(u) \tau_{y} \varrho d x-\varepsilon \int_{\mathbb{R}^{n}}|\nabla u|^{2} \tau_{y} \varrho d x \\
& \quad-\int_{\mathbb{R}^{n}} u_{t} \nabla u \cdot \nabla \tau_{y} \varrho d x-\varepsilon \int_{\mathbb{R}^{n}} u \nabla u \cdot \nabla \tau_{y} \varrho d x+\int_{\mathbb{R}^{n}} g\left(u_{t}+\varepsilon u\right) \tau_{y} \varrho d x .
\end{aligned}
$$

Thanks to (4) and (15) the right hand side of (18) is, for small $\delta>0$, dominated by

$$
\begin{aligned}
\varepsilon\left[-\frac{\eta-\varepsilon}{2 \varepsilon} \int_{\mathbb{R}^{n}} u_{t}^{2} \tau_{y} \varrho d x-\right. & \frac{\mu}{2} \int_{\mathbb{R}^{n}} u^{2} \tau_{y} \varrho d x \\
& \left.-\frac{1}{2} \int_{\mathbb{R}^{n}}|\nabla u|^{2} \tau_{y} \varrho d x+k \int_{\mathbb{R}^{n}} F(u) \tau_{y} \varrho d x\right] \\
& +\left(\frac{\varepsilon}{\mu}+\frac{1}{\eta-\varepsilon}\right) \int_{\mathbb{R}^{n}} g^{2} \tau_{y} \varrho d x+C_{\mu} \varepsilon \int_{\mathbb{R}^{n}} \tau_{y} \varrho d x .
\end{aligned}
$$

Choosing $\mu=\mu_{0}=2 \varepsilon(\eta-\varepsilon)=\frac{\eta-\varepsilon}{\varepsilon} \varepsilon^{2}+\varepsilon(\eta-\varepsilon)$ we dominate the last expression by

$$
\begin{aligned}
\varepsilon\left[-\int_{\mathbb{R}^{n}} u_{t}^{2} \tau_{y} \varrho\right. & d x-\varepsilon^{2} \int_{\mathbb{R}^{n}} u^{2} \tau_{y} \varrho d x-\frac{1}{2} \varepsilon(\eta-\varepsilon) \int_{\mathbb{R}^{n}} u^{2} \tau_{y} \varrho d x \\
& \left.-\frac{1}{2} \int_{\mathbb{R}^{n}}|\nabla u|^{2} \tau_{y} \varrho d x+\int_{\mathbb{R}^{n}} F(u) \tau_{y} \varrho d x\right]+\frac{3}{2(\eta-\varepsilon)} \int_{\mathbb{R}^{n}} g^{2} \tau_{y} \varrho d x \\
& +(k-1) \varepsilon \int_{\mathbb{R}^{n}} F(u) \tau_{y} \varrho d x+C_{\mu_{0}} \varepsilon \int_{\mathbb{R}^{n}} \tau_{y} \varrho d x .
\end{aligned}
$$


Since

$$
-\int_{\mathbb{R}^{n}} u_{t}^{2} \tau_{y} \varrho d x-\varepsilon^{2} \int_{\mathbb{R}^{n}} u^{2} \tau_{y} \varrho d x \leq-\frac{1}{2} \int_{\mathbb{R}^{n}}\left(u_{t}+\varepsilon u\right)^{2} \tau_{y} \varrho d x
$$

and

$$
(k-1) \varepsilon \int_{\mathbb{R}^{n}} F(u) \tau_{y} \varrho d x \leq(k-1) \varepsilon C_{\nu}^{\prime} \int \tau_{y} \varrho d x,
$$

we next estimate the right hand side of (18) by

$$
-\varepsilon \mathcal{L}\left(u, u_{t}\right)+\frac{3}{2(\eta-\varepsilon)} \int_{\mathbb{R}^{n}} g^{2} \tau_{y} \varrho d x+\varepsilon\left(C_{\mu_{0}}+(k-1) C_{\nu}^{\prime}\right) \int_{\mathbb{R}^{n}} \tau_{y} \varrho d x .
$$

It is thus seen that

$$
\frac{d}{d t} \mathcal{L}\left(u, u_{t}\right) \leq-\varepsilon \mathcal{L}\left(u, u_{t}\right)+\varepsilon C_{1},
$$

and hence

$$
\mathcal{L}\left(u, u_{t}\right) \leq \mathcal{L}\left(u_{0}, v_{0}\right) e^{-\varepsilon t}+C_{1}\left(1-e^{-\varepsilon t}\right)
$$

where

$$
C_{1}=\frac{3}{\mu_{0}} \sup _{y \in \mathbb{R}^{n}} \int_{\mathbb{R}^{n}} g^{2} \tau_{y} \varrho d x+\left(C_{\mu_{0}}+(k-1) C_{\nu}^{\prime}\right) \int_{\mathbb{R}^{n}} \varrho d x .
$$

Thanks to (16), for three components of the integrand in $\mathcal{L}\left(u, u_{t}\right)$, we have $\frac{1}{2}\left(u_{t}+\varepsilon u\right)^{2}+\frac{1}{2} \varepsilon(\eta-\varepsilon) u^{2}-F(u) \geq \frac{1}{2}(1-\varepsilon) u_{t}^{2}+\frac{1}{2}[\varepsilon(\eta-1)+2 \nu] u^{2}-C_{\nu}^{\prime}$. Choosing now $\varepsilon$ sufficiently small satisfying (17) and $\nu=\nu_{1}$ we can achieve

$$
\nu_{0}:=\min \left\{1-\varepsilon, \varepsilon(\eta-1)+2 \nu_{1}\right\}>0,
$$

which leads to the estimate

$$
\mathcal{L}\left(u, u_{t}\right) \geq \frac{\nu_{0}}{2} \int_{\mathbb{R}^{n}}\left(u_{t}^{2}+u^{2}\right) \tau_{y} \varrho d x+\frac{\nu_{0}}{2} \int_{\mathbb{R}^{n}}|\nabla u|^{2} \tau_{y} \varrho d x-C_{2}
$$

with $C_{2}=C_{\nu_{1}}^{\prime} \int_{\mathbb{R}^{n}} \varrho d x$. Combining (20) and (21) we get

$$
\frac{\nu_{0}}{2} \int_{\mathbb{R}^{n}}\left(u_{t}^{2}+u^{2}+|\nabla u|^{2}\right) \tau_{y} \varrho d x \leq \mathcal{L}\left(u_{0}, v_{0}\right) e^{-\varepsilon t}+C_{1}\left(1-e^{-\varepsilon t}\right)+C_{2} .
$$

Estimating the term $\left|\int_{\mathbb{R}^{n}} F\left(u_{0}\right) \tau_{y} \varrho d x\right|$ by $\operatorname{const}\left(\left\|u_{0}\right\|_{\dot{H}_{\mathrm{lu}}^{1}\left(\mathbb{R}^{n}\right)}^{q+1}+\int_{\mathbb{R}^{n}} \tau_{y} \varrho d x\right)$ we finally obtain

$$
\frac{\nu_{0}}{2} \int_{\mathbb{R}^{n}}\left(u_{t}^{2}+u^{2}+|\nabla u|^{2}\right) \tau_{y} \varrho d x \leq \operatorname{const}\left(\left\|u_{0}\right\|_{\dot{H}_{\mathrm{lu}}^{1}\left(\mathbb{R}^{n}\right)},\left\|v_{0}\right\|_{\dot{L}_{\mathrm{lu}}^{2}\left(\mathbb{R}^{n}\right)},\|g\|_{\dot{L}_{\mathrm{lu}}^{2}\left(\mathbb{R}^{n}\right)}\right),
$$

and

$$
\limsup _{t \rightarrow \infty} \frac{\nu_{0}}{2} \int_{\mathbb{R}^{n}}\left(u_{t}^{2}+u^{2}+|\nabla u|^{2}\right) \tau_{y} \varrho d x \leq C_{1}+C_{2}
$$


This leads to the estimate

$$
\begin{aligned}
\left\|\left(u, u_{t}\right)\right\|_{\dot{H}_{\mathrm{lu}}^{1}\left(\mathbb{R}^{n}\right) \times \dot{L}_{\mathrm{lu}}^{2}\left(\mathbb{R}^{n}\right)}^{2} & \\
\leq & \frac{6}{\nu_{0}} \operatorname{const}\left(\left\|u_{0}\right\|_{\dot{H}_{\mathrm{lu}}^{1}\left(\mathbb{R}^{n}\right)},\left\|v_{0}\right\|_{\dot{L}_{\mathrm{lu}}^{2}\left(\mathbb{R}^{n}\right)},\|g\|_{\dot{L}_{\mathrm{lu}}^{2}\left(\mathbb{R}^{n}\right)}\right)
\end{aligned}
$$

and to the asymptotic estimate

$$
\limsup _{t \rightarrow \infty}\left\|\left(u, u_{t}\right)\right\|_{\dot{H}_{\mathrm{lu}}^{1}\left(\mathbb{R}^{n}\right) \times \dot{L}_{\mathrm{lu}}^{2}\left(\mathbb{R}^{n}\right)}^{2} \leq \frac{6}{\nu_{0}}\left(C_{1}+C_{2}\right) .
$$

TheOREM 3.1. Suppose $n \leq 3$, (13)-(16) hold, $\eta>0$ and $g \in \dot{L}_{\mathrm{lu}}^{2}\left(\mathbb{R}^{n}\right)$. Then the problem (10) defines in $X=\dot{H}_{\mathrm{lu}}^{1}\left(\mathbb{R}^{n}\right) \times \dot{L}_{\mathrm{lu}}^{2}\left(\mathbb{R}^{n}\right)$ a $C^{0}$ semigroup $\{T(t)\}$ of global mild solutions which has bounded orbits of bounded sets and possesses a bounded absorbing set.

Proof. In the light of our previous considerations we merely need to justify the uniform continuity of the solution on bounded time intervals with respect to the initial condition. However, since orbits of bounded sets are bounded and the nonlinear term in (10) is Lipschitz continuous on bounded sets from $X$ to $X$, this property is a direct consequence of (11) and the Gronwall inequality.

3.2. Globally bounded smooth solutions to (8). Our further concern is smooth $\dot{H}_{\mathrm{lu}}^{2}\left(\mathbb{R}^{n}\right) \times \dot{H}_{\mathrm{lu}}^{1}\left(\mathbb{R}^{n}\right)$ solutions to (10) for $n \leq 3$. To get uniform in time estimates of their $\dot{H}_{\mathrm{lu}}^{2}\left(\mathbb{R}^{n}\right) \times \dot{H}_{\mathrm{lu}}^{1}\left(\mathbb{R}^{n}\right)$-norm and construct a global attractor we need to sharpen the growth restriction imposed on $f^{\prime}$ in conditions (13), (14). Assume, from now on, that the nonlinear term $f$ has a subcritical growth, that is,

$$
\left|f^{\prime}(s)\right| \leq c\left(1+|s|^{2-\delta_{0}}\right) \quad \text { with } 0<\delta_{0}<1 \text { when } n=3 .
$$

Formal time differentiation of (8) leads to the Cauchy problem, for $v=$ $u_{t}$, having the form

$$
\left\{\begin{array}{l}
v_{t t}+\eta v_{t}-\Delta v=f^{\prime}(u) v, \quad t>0, x \in \mathbb{R}^{n}, \\
v(0, x)=v_{0}(x), \quad v_{t}(0, x)=w_{0}(x), \quad x \in \mathbb{R}^{n} .
\end{array}\right.
$$

Since the linear semigroup $\{\mathcal{T}(t)\}$ introduced in Theorem 2.1 does not decay to zero as $t \rightarrow \infty$, consider

$$
\begin{aligned}
& \frac{d}{d t}\left[\begin{array}{c}
v \\
w
\end{array}\right]=\left(A_{\eta}+\left[\begin{array}{cc}
0 & 0 \\
-\gamma I & 0
\end{array}\right]\right)\left[\begin{array}{c}
v \\
w
\end{array}\right]+\left[\begin{array}{c}
0 \\
f^{\prime}(u) v+\gamma v
\end{array}\right], \quad \gamma>0, \\
& {\left[\begin{array}{c}
v \\
w
\end{array}\right]_{t=0}=\left[\begin{array}{c}
v_{0} \\
w_{0}
\end{array}\right] \in X }
\end{aligned}
$$


and the corresponding integral formula

$$
\begin{aligned}
{\left[\begin{array}{c}
v \\
w
\end{array}\right]=} & \mathcal{S}(t)\left[\begin{array}{c}
v_{0} \\
w_{0}
\end{array}\right] \\
& +\int_{0}^{t} \mathcal{S}(t-s)\left[\begin{array}{c}
0 \\
f^{\prime}(u(s)) v(s)+\gamma v(s)
\end{array}\right] d s, \quad t \geq 0,\left[\begin{array}{c}
v_{0} \\
w_{0}
\end{array}\right] \in X,
\end{aligned}
$$
where $\{\mathcal{S}(t)\}$ denotes the linear semigroup generated by $A_{\eta}+\left[\begin{array}{cc}0 & 0 \\ -\gamma I & 0\end{array}\right]$
on $X$.

Note that, for $\left[\begin{array}{l}u_{0} \\ v_{0}\end{array}\right] \in \dot{H}_{\mathrm{lu}}^{2}\left(\mathbb{R}^{n}\right) \times \dot{H}_{\mathrm{lu}}^{1}\left(\mathbb{R}^{n}\right)$, the first coordinate of $\left\{\mathcal{S}(t)\left[\begin{array}{l}u_{0} \\ v_{0}\end{array}\right]\right\}$ is a solution to

$$
\left\{\begin{array}{l}
\omega_{t t}+\eta \omega_{t}-\Delta \omega=-\gamma \omega, \quad t>0, x \in \mathbb{R}^{n} \\
\omega(0, x)=u_{0}(x), \quad \omega_{t}(0, x)=v_{0}(x), \quad x \in \mathbb{R}^{n}
\end{array}\right.
$$

Therefore, estimating as in Subsection 3.1 and using a density argument we obtain

$$
\begin{aligned}
\left\|\mathcal{S}(t)\left[\begin{array}{c}
u_{0} \\
v_{0}
\end{array}\right]\right\|_{\dot{H}_{\mathrm{lu}}^{1}\left(\mathbb{R}^{n}\right) \times \dot{L}_{\mathrm{lu}}^{2}\left(\mathbb{R}^{n}\right)} \\
\quad \leq \mathcal{C}\left\|\left[\begin{array}{l}
u_{0} \\
v_{0}
\end{array}\right]\right\|_{\dot{H}_{\mathrm{lu}}^{1}\left(\mathbb{R}^{n}\right) \times \dot{L}_{\mathrm{lu}}^{2}\left(\mathbb{R}^{n}\right)} e^{-\varepsilon t}, \quad t \geq 0,\left[\begin{array}{l}
u_{0} \\
v_{0}
\end{array}\right] \in X .
\end{aligned}
$$

We are now ready to prove

LEMma 3.2. Under (24) and the assumptions of Theorem 3.1, for any bounded set $B \subset \dot{H}_{\mathrm{lu}}^{2}\left(\mathbb{R}^{n}\right) \times \dot{H}_{\mathrm{lu}}^{1}\left(\mathbb{R}^{n}\right)$ there exists $M_{B}>0$ such that

$$
\left\|\left[\begin{array}{l}
u\left(t, u_{0}, v_{0}\right) \\
v\left(t, u_{0}, v_{0}\right)
\end{array}\right]\right\|_{\dot{H}_{\mathrm{lu}}^{2}\left(\mathbb{R}^{n}\right) \times \dot{H}_{\mathrm{lu}}^{1}\left(\mathbb{R}^{n}\right)} \leq M_{B} \quad \text { for each } t>0,\left[\begin{array}{l}
u_{0} \\
v_{0}
\end{array}\right] \in B .
$$

Proof. Let us focus on the case $n=3$. Since $\left[\begin{array}{l}u_{0} \\ v_{0}\end{array}\right]$ belongs to a bounded subset $B$ of $D\left(A_{\eta}\right)=\dot{H}_{\mathrm{lu}}^{2}\left(\mathbb{R}^{n}\right) \times \dot{H}_{\mathrm{lu}}^{1}\left(\mathbb{R}^{n}\right)$ and $T(t)\left(\left[\begin{array}{l}u_{0} \\ v_{0}\end{array}\right]\right)$ is a classical solution to (10) the quantity

$$
w_{0}:=u_{t t}(0)=-\eta v_{0}+\Delta u_{0}+f\left(u_{0}\right)+g \in \dot{L}_{\mathrm{lu}}^{2}\left(\mathbb{R}^{n}\right)
$$

is well defined. Also, the elements $\left[\begin{array}{c}v_{0} \\ w_{0}\end{array}\right]$ remain in a certain set $\tilde{B}$, bounded in $\dot{H}_{\mathrm{lu}}^{1}\left(\mathbb{R}^{n}\right) \times \dot{L}_{\mathrm{lu}}^{2}\left(\mathbb{R}^{n}\right)$, and the function

satisfies (26).

$$
\left[\begin{array}{c}
v(t) \\
w(t)
\end{array}\right]=\frac{d}{d t} T(t)\left(\left[\begin{array}{l}
u_{0} \\
v_{0}
\end{array}\right]\right)
$$


Furthermore, the linear semigroup in (26) decays exponentially to zero and, thanks to (24), we obtain a subordination condition of the form

$$
\begin{aligned}
& \left\|\left[\begin{array}{c}
0 \\
f^{\prime}(u(s)) v(s)+\gamma v(s)
\end{array}\right]\right\|_{\dot{H}_{\mathrm{lu}}^{1}\left(\mathbb{R}^{n}\right) \times \dot{L}_{\mathrm{lu}}^{2}\left(\mathbb{R}^{n}\right)} \\
& \leq\left\|f^{\prime}(u(s)) v(s)\right\|_{\dot{L}_{\mathrm{lu}}^{2}\left(\mathbb{R}^{n}\right)}+\gamma\|v(s)\|_{\dot{L}_{\mathrm{lu}}^{2}\left(\mathbb{R}^{n}\right)} \\
& \leq c\left\|1+|u(s)|^{2-\delta_{0}}\right\|_{\dot{L}_{\mathrm{lu}}^{3+\delta_{1}}\left(\mathbb{R}^{n}\right)}\|v(s)\|_{\dot{L}_{\mathrm{lu}}^{6-\delta_{2}}\left(\mathbb{R}^{n}\right)}+\gamma\|v(s)\|_{\dot{L}_{\mathrm{lu}}^{2}\left(\mathbb{R}^{n}\right)} \\
& \leq G\left(\|u(s)\|_{\dot{H}_{\mathrm{lu}}^{1}\left(\mathbb{R}^{n}\right)}\right)\|v(s)\|_{\dot{L}_{\mathrm{lu}}^{2}\left(\mathbb{R}^{n}\right)}^{1-\theta}\|v(s)\|_{\dot{H}_{\mathrm{lu}}^{1}\left(\mathbb{R}^{n}\right)}^{\theta}+\gamma\|v(s)\|_{\dot{L}_{\mathrm{lu}}^{2}\left(\mathbb{R}^{n}\right)} \\
& \leq\left[G\left(\|u(s)\|_{\dot{H}_{\mathrm{lu}}^{1}\left(\mathbb{R}^{n}\right)}\right)+\gamma\right]\|v(s)\|_{\dot{L}_{\mathrm{lu}}^{2}\left(\mathbb{R}^{n}\right)}^{1-\theta}\|v(s)\|_{\dot{H}_{\mathrm{lu}}^{1}\left(\mathbb{R}^{n}\right)}^{\theta} \\
& \leq G_{1}\left(\|u(s)\|_{\dot{H}_{\mathrm{lu}}^{1}\left(\mathbb{R}^{n}\right)}+\|v(s)\|_{\dot{L}_{\mathrm{lu}}^{2}\left(\mathbb{R}^{n}\right)}\right)\left\|\left[\begin{array}{c}
v(s) \\
w(s)
\end{array}\right]\right\|_{\dot{H}_{\mathrm{lu}}^{1}\left(\mathbb{R}^{n}\right) \times \dot{L}_{\mathrm{lu}}^{2}\left(\mathbb{R}^{n}\right)}^{\theta},
\end{aligned}
$$

where $G, G_{1}:[0, \infty) \rightarrow[0, \infty)$ are nondecreasing continuous functions and $\theta \in(0,1)$.

Since a uniform (in $s \geq 0$ ) estimate of $\|u(s)\|_{\dot{H}_{\mathrm{lu}}^{1}\left(\mathbb{R}^{n}\right)}+\|v(s)\|_{\dot{L}_{\mathrm{lu}}^{2}\left(\mathbb{R}^{n}\right)}$ has already been found in Subsection 3.1, we have

$$
\begin{aligned}
\left\|\left[\begin{array}{c}
v(t) \\
w(t)
\end{array}\right]\right\|_{\dot{H}_{\mathrm{lu}}^{1}\left(\mathbb{R}^{n}\right) \times \dot{L}_{\mathrm{lu}}^{2}\left(\mathbb{R}^{n}\right)} \leq \mathcal{C}\left\|\left[\begin{array}{c}
v_{0} \\
w_{0}
\end{array}\right]\right\|_{\dot{H}_{\mathrm{lu}}^{1}\left(\mathbb{R}^{n}\right) \times \dot{L}_{\mathrm{lu}}^{2}\left(\mathbb{R}^{n}\right)} e^{-\varepsilon t} \\
+\quad+\mathcal{C} \int_{0}^{t} e^{-\varepsilon(t-s)}\left\|\left[\begin{array}{c}
0 \\
f^{\prime}(u(s)) v(s)+\gamma v(s)
\end{array}\right]\right\|_{\dot{H}_{\mathrm{lu}}^{1}\left(\mathbb{R}^{n}\right) \times \dot{L}_{\mathrm{lu}}^{2}\left(\mathbb{R}^{n}\right)} d s \\
\leq \mathcal{C}\left\|\left[\begin{array}{c}
v_{0} \\
w_{0}
\end{array}\right]\right\|_{\dot{H}_{\mathrm{lu}}^{1}\left(\mathbb{R}^{n}\right) \times \dot{L}_{\mathrm{lu}}^{2}\left(\mathbb{R}^{n}\right)} e^{-\varepsilon t} \\
+\mathcal{C} G_{1}\left(\sup _{s \in[0, t]}\left\{\|u(s)\|_{\dot{H}_{\mathrm{lu}}^{1}\left(\mathbb{R}^{n}\right)}+\|v(s)\|_{\dot{L}_{\mathrm{lu}}^{2}\left(\mathbb{R}^{n}\right)}\right\}\right) \\
\quad \times \sup _{s \in[0, t]}\left\|\left[\begin{array}{c}
v(s) \\
w(s)
\end{array}\right]\right\|_{\dot{H}_{\mathrm{lu}}^{1}\left(\mathbb{R}^{n}\right) \times \dot{L}_{\mathrm{lu}}^{2}\left(\mathbb{R}^{n}\right)}^{\theta}
\end{aligned}
$$

Therefore the function

$$
z(t)=\sup _{s \in[0, t]}\left\|\left[\begin{array}{c}
v(s) \\
w(s)
\end{array}\right]\right\|_{\dot{H}_{1 \mathrm{u}}^{1}\left(\mathbb{R}^{n}\right) \times \dot{L}_{1 \mathrm{lu}}^{2}\left(\mathbb{R}^{n}\right)}
$$

satisfies

$$
z(t) \leq C\left(1+z(t)^{\theta}\right), \quad t>0,
$$

and hence

$$
\sup _{t \geq 0}\left\|\left[\begin{array}{c}
v(t) \\
w(t)
\end{array}\right]\right\|_{\dot{H}_{\mathrm{lu}}^{1}\left(\mathbb{R}^{n}\right) \times \dot{L}_{\mathrm{lu}}^{2}\left(\mathbb{R}^{n}\right)} \leq z_{1},
$$


where $z_{1}$ is the unique positive root of the equation $z=C\left(1+z^{\theta}\right)$ (see [C-D 1, Theorem 3.1.1] for details).

Estimating $\Delta u$ with the aid of (8) we obtain (for $w=u_{t t}, v=u_{t}$ )

$$
\|\Delta u\|_{\dot{L}_{\mathrm{lu}}^{2}\left(\mathbb{R}^{n}\right)} \leq\|w\|_{\dot{L}_{\mathrm{lu}}^{2}\left(\mathbb{R}^{n}\right)}+\eta\|v\|_{\dot{L}_{\mathrm{lu}}^{2}\left(\mathbb{R}^{n}\right)}+\|f(u)\|_{\dot{L}_{\mathrm{lu}}^{2}\left(\mathbb{R}^{n}\right)}+\|g\|_{\dot{L}_{\mathrm{lu}}^{2}\left(\mathbb{R}^{n}\right)},
$$

where all the terms appearing on the right hand side have already been estimated independently of $t \geq 0$ and $\left(u_{0}, v_{0}\right) \in B$. Since $-\Delta+\lambda I(\lambda>0)$ is an isomorphism of $\dot{H}_{\text {lu }}^{2}\left(\mathbb{R}^{n}\right)$ onto $\dot{L}_{\text {lu }}^{2}\left(\mathbb{R}^{n}\right)$ (see [A-C-D-RB, Theorems 5.2 and 5.3]) it is clear that (28) and the previously obtained bound on $u$ yield an $\dot{H}_{\text {lu }}^{2}\left(\mathbb{R}^{n}\right)$-estimate of $u$. The proof is complete.

REMARK 3.3. When the growth of $f$ is critical, that is, $f$ is cubic if $n=3$, a similar procedure will still give a bound on the solutions in $D\left(A_{\eta}\right)$, but uniform merely on bounded time intervals.

According to (23) the $\dot{H}_{\text {lu }}^{1}\left(\mathbb{R}^{n}\right) \times \dot{L}_{\text {lu }}^{2}\left(\mathbb{R}^{n}\right)$-estimate is asymptotically independent of $\left(u_{0}, v_{0}\right)$ varying in bounded subsets of $\dot{H}_{\mathrm{lu}}^{1}\left(\mathbb{R}^{n}\right) \times \dot{L}_{\mathrm{lu}}^{2}\left(\mathbb{R}^{n}\right)$. As in [C-D 1, Corollary 4.1.3] we thus conclude that

Lemma 3.4. Under the assumptions of Lemma 3.2 there is a set $\mathcal{B}_{0}$ bounded in $D\left(A_{\eta}\right)=\dot{H}_{\text {lu }}^{2}\left(\mathbb{R}^{n}\right) \times \dot{H}_{\mathrm{lu}}^{1}\left(\mathbb{R}^{n}\right)$ and absorbing bounded subsets of $D\left(A_{\eta}\right)$ in $D\left(A_{\eta}\right)$ norm.

Proof. Note that

$$
\left\|\left[\begin{array}{c}
v(t) \\
w(t)
\end{array}\right]\right\|_{\dot{H}_{1 \mathrm{lu}}^{1}\left(\mathbb{R}^{n}\right) \times \dot{L}_{\mathrm{lu}}^{2}\left(\mathbb{R}^{n}\right)}
$$

remains asymptotically independent of $\left(v_{0}, w_{0}\right)$ (see [C-D 2, Theorem 1] for details). From (28) and (23) the same is true for $\|\Delta u\|_{\dot{L}_{\mathrm{lu}}^{2}\left(\mathbb{R}^{n}\right)}$. This may be finally translated to an asymptotic estimate of $\|u\|_{\dot{H}_{\mathrm{lu}}^{2}\left(\mathbb{R}^{n}\right)}$ with the aid of the isomorphism property of $-\Delta+\lambda I(\lambda>0)$.

4. Continuity and asymptotic compactness of $\{T(t)\}$. In the following two subsections we will check additional conditions necessary to deduce the existence of a global attractor for space dimensions $n \leq 3$.

4.1. Weak asymptotic compactness property. Recall from [CA-DL], [A-C-D-RB] that any sequence bounded in $H_{\mathrm{lu}}^{2}\left(\mathbb{R}^{n}\right) \times H_{\mathrm{lu}}^{1}\left(\mathbb{R}^{n}\right)$ is precompact in $X_{\varrho}=H_{\varrho}^{1}\left(\mathbb{R}^{n}\right) \times L_{\varrho}^{2}\left(\mathbb{R}^{n}\right)$. In addition, each element obtained as a limit of its subsequence in $X_{\varrho}$-norm must in fact belong to $\dot{H}_{\text {lu }}^{1}\left(\mathbb{R}^{n}\right) \times \dot{L}_{\text {lu }}^{2}\left(\mathbb{R}^{n}\right)$. This leads to the following conclusion.

LemMA 4.1. Suppose that $n \leq 3$ and the assumptions of Lemma 3.2 hold, and let $E=\operatorname{cl}_{X_{\varrho}}\left(\gamma^{+}\left(\mathcal{B}_{0}\right)\right)$. Then $E$ is bounded in $X$ and the semigroup 
$\{T(t)\}$ is asymptotically compact on $E$, i.e. if $\left\{\left(u_{0 m}, v_{0 m}\right)\right\}$ is contained in $E$ and $t_{m} \rightarrow \infty$, then $\left\{T\left(t_{m}\right)\left[\begin{array}{l}u_{0 m} \\ v_{0 m}\end{array}\right]\right\}$ contains a subsequence convergent in $X_{\varrho}$-norm to an element of $E$.

Proof. Thanks to boundedness of $\gamma^{+}\left(\mathcal{B}_{0}\right)$ in $\dot{H}_{\mathrm{lu}}^{2}\left(\mathbb{R}^{n}\right) \times \dot{H}_{\mathrm{lu}}^{1}\left(\mathbb{R}^{n}\right)$, the sequences from $\gamma^{+}\left(\mathcal{B}_{0}\right)$ approximating limit points $(\phi, \psi) \in \operatorname{cl}_{X_{\varrho}}\left(\gamma^{+}\left(\mathcal{B}_{0}\right)\right)$ in $X_{\varrho}$ will be bounded in the norm of $\dot{H}_{\mathrm{lu}}^{2}\left(\mathbb{R}^{n}\right) \times \dot{H}_{\mathrm{lu}}^{1}\left(\mathbb{R}^{n}\right)$. Thus, $\mathrm{cl}_{X_{\varrho}}\left(\gamma^{+}\left(\mathcal{B}_{0}\right)\right)$ is in fact bounded in $X$.

If $t_{m} \rightarrow \infty$ and $\left\{\left(u_{0 m}, v_{0 m}\right)\right\}$ is contained in $E$ then, since $E$ is positively invariant (see (29) below), $\left\{T\left(t_{m}\right)\left(\left[\begin{array}{l}u_{0 m} \\ v_{0 m}\end{array}\right]\right)\right\}$ remains in $E$. Therefore, there exists $\left\{\left[\begin{array}{l}\phi_{m} \\ \psi_{m}\end{array}\right]\right\} \subset \gamma^{+}\left(\mathcal{B}_{0}\right)$ such that

$$
\left\|T\left(t_{m}\right)\left(\left[\begin{array}{l}
u_{0 m} \\
v_{0 m}
\end{array}\right]\right)-\left[\begin{array}{l}
\phi_{m} \\
\psi_{m}
\end{array}\right]\right\|_{X_{\varrho}} \leq \frac{1}{m}, \quad m \in \mathbb{N} .
$$

Since $\left\{\left[\begin{array}{l}\phi_{m} \\ \psi_{m}\end{array}\right]\right\}$ is bounded in $D\left(A_{\eta}\right)$, there exists a subsequence $\left\{\left[\begin{array}{l}\phi_{m_{k}} \\ \psi_{m_{k}}\end{array}\right]\right\}$ convergent in $X_{\varrho}$ to a certain $\left[\begin{array}{c}\bar{\phi} \\ \bar{\psi}\end{array}\right] \in X$. The above implies that

$$
\begin{aligned}
\| T\left(t_{m_{k}}\right) & \left(\left[\begin{array}{l}
u_{0 m_{k}} \\
v_{0 m_{k}}
\end{array}\right]\right)-\left[\begin{array}{c}
\bar{\phi} \\
\bar{\psi}
\end{array}\right] \|_{X_{\varrho}} \\
& \leq\left\|T\left(t_{m_{k}}\right)\left(\left[\begin{array}{l}
u_{0 m_{k}} \\
v_{0 m_{k}}
\end{array}\right]\right)-\left[\begin{array}{l}
\phi_{m_{k}} \\
\psi_{m_{k}}
\end{array}\right]\right\|_{X_{\varrho}}+\left\|\left[\begin{array}{l}
\phi_{m_{k}} \\
\psi_{m_{k}}
\end{array}\right]-\left[\begin{array}{c}
\bar{\phi} \\
\bar{\psi}
\end{array}\right]\right\|_{X_{\varrho}},
\end{aligned}
$$

with the right hand side convergent to zero as $k \rightarrow \infty$. Evidently $(\bar{\phi}, \bar{\psi})$ belongs to $E$, which completes the proof.

4.2. Continuity in $X_{\varrho}$. For the set $E=\operatorname{cl}_{X_{\varrho}}\left(\gamma^{+}\left(\mathcal{B}_{0}\right)\right)$ we will now show the continuity of the semigroup $T(t): E \rightarrow E, t \geq 0$; namely

$$
\begin{aligned}
& \text { if }\left\{\left(u_{0 m}, v_{0 m}\right)\right\} \subset E \text { and }\left(u_{0 m}, v_{0 m}\right) \stackrel{X_{\varrho}}{\rightarrow}\left(u_{0}, v_{0}\right) \in E, \\
& \text { then } T(t)\left(u_{0 m}, v_{0 m}\right) \stackrel{X_{\varrho}}{\longrightarrow} T(t)\left(u_{0}, v_{0}\right) \in E \text { for each } t \geq 0 .
\end{aligned}
$$

Note first that the set $\gamma^{+}\left(\mathcal{B}_{0}\right)$ is bounded in $D\left(A_{\eta}\right)$. Then, thanks to the embedding $H_{\mathrm{lu}}^{2}\left(\mathbb{R}^{n}\right) \subset L^{\infty}\left(\mathbb{R}^{n}\right), n \leq 3$, the first coordinate $\phi$ of any pair $(\phi, \psi) \in \gamma^{+}\left(\mathcal{B}_{0}\right)$ stays in a bounded subset of $L^{\infty}\left(\mathbb{R}^{n}\right)$. Therefore,

$$
\exists_{M>0} \forall_{(\phi, \psi) \in \operatorname{conv}\left(\gamma^{+}\left(\mathcal{B}_{0}\right)\right)}\left|f^{\prime}(\phi)\right| \leq M .
$$


Now, for any $\left[\begin{array}{l}u_{1} \\ v_{1}\end{array}\right],\left[\begin{array}{l}u_{2} \\ v_{2}\end{array}\right] \in \gamma^{+}\left(\mathcal{B}_{0}\right)$ the $X_{\varrho}$ norm of $T(t)\left(\left[\begin{array}{l}u_{1}-u_{2} \\ v_{1}-v_{2}\end{array}\right]\right)$ will be estimated in terms of $\left\|\left[\begin{array}{c}u_{1}-u_{2} \\ v_{1}-v_{2}\end{array}\right]\right\|_{X_{\varrho}}$. Indeed, setting $U=u_{1}-u_{2}$, we find that $U$ satisfies

$$
U_{t t}+\eta U_{t}-\Delta U=f\left(u_{1}\right)-f\left(u_{2}\right) .
$$

Multiplying (31) by $\left(U_{t}+\varepsilon U\right) \varrho, 0<\varepsilon<(\eta-\varepsilon) / 4$, and calculating as in Subsection 3.1 we find that $\mathcal{H}\left(U, U_{t}\right):=\int_{\mathbb{R}^{n}}\left[\frac{1}{2}\left(U_{t}+\varepsilon U\right)^{2}+\frac{1}{2} \varepsilon(\eta-\varepsilon) U^{2}+\right.$ $\left.\frac{1}{2}|\nabla U|^{2}\right] \varrho d x$ satisfies

$$
\frac{d}{d t} \mathcal{H}\left(U, U_{t}\right) \leq \text { const } \mathcal{H}\left(U, U_{t}\right)
$$

and hence

$$
\begin{aligned}
\frac{1}{4}\left\|\left(U(t), U_{t}(t)\right)\right\|_{X_{\varrho}}^{2} & \leq \mathcal{H}\left(U(t), U_{t}(t)\right) \leq \mathcal{H}\left(U(0), U_{t}(0)\right) e^{\text {const } t} \\
& \leq \operatorname{const}_{1}\left\|\left(U(0), U_{t}(0)\right)\right\|_{X_{\varrho}}^{2} .
\end{aligned}
$$

By a density argument, estimate (32) holds for $U$ being the difference of any two elements of $E$, which proves (29). Note that the limit function $T(t)\left(u_{0}, v_{0}\right)$ obtained in (29) is in fact a mild solution to (10). This may be verified by letting $m \rightarrow \infty$ in the integral formula

$$
\left[\begin{array}{l}
u_{m}(t) \\
v_{m}(t)
\end{array}\right]=\mathcal{T}(t)\left[\begin{array}{l}
u_{0 m} \\
v_{0 m}
\end{array}\right]+\int_{0}^{t} \mathcal{T}(t-s)\left(\left[\begin{array}{c}
0 \\
f\left(u_{m}(s)\right)+g
\end{array}\right]\right) d s, \quad t \geq 0
$$

defining a mild solution for smooth data $\left(u_{0 m}, v_{0 m}\right) \in \gamma^{+}\left(\mathcal{B}_{0}\right)$.

The above considerations show the validity of all assumptions in the theorem of [H, p. 39] concerning the existence of a global attractor. Under the assumptions of Lemma 4.1 we thus conclude that:

THEOREM 4.2. The semigroup $\{T(t)\}$ restricted to a complete metric subspace $E$ of $X_{\varrho}$ has a global attractor $\mathcal{A}$. The attractor $\mathcal{A}$ is moreover bounded in $X$-norm, attracts bounded subsets of $\dot{H}_{\mathrm{lu}}^{2}\left(\mathbb{R}^{n}\right) \times \dot{H}_{\mathrm{lu}}^{1}\left(\mathbb{R}^{n}\right)$ in the topology of $X_{\varrho}$ and is invariant with respect to the group of translations in $\mathbb{R}^{n}$.

REMARK 4.3. Note that the assumptions of [F, condition (1.2)] in our notation read

$$
f \in C^{1}(\mathbb{R}), \quad \limsup _{|s| \rightarrow \infty} \frac{f(s)}{s}<0, \quad \exists_{M>0} \forall s \in \mathbb{R} f^{\prime}(s) \leq M .
$$

Also, $f(s)=s-s^{3}+2 s \cos s^{2}$ is an example of a nonlinearity satisfying (15), (16) for which the last condition in (34) fails. In particular such an $f$ will not be covered by the results of $[\mathrm{F}]$. 


\section{References}

[A-C-D-RB] J. Arrieta, J. W. Cholewa, T. Dlotko and A. Rodriguez-Bernal, Linear parabolic equations in locally uniform spaces, Math. Models Methods Appl. Sci. 14 (2004), 253-293.

[CA-DL] A. N. Carvalho and T. Dlotko, Partly dissipative systems in uniformly local spaces, Colloq. Math. 100 (2004), 221-242.

[C-D 1] J. W. Cholewa and T. Dlotko, Global Attractors in Abstract Parabolic Problems, Cambridge Univ. Press, Cambridge, 2000.

[C-D 2] -, -, Cauchy problem with subcritical nonlinearity, J. Math. Anal. Appl. 210 (1997), 531-548.

[F] E. Feireisl, Bounded, locally compact global attractors for semilinear damped wave equations in $\mathbb{R}^{n}$, Differential Integral Equations 9 (1996), 1147-1156.

[H] J. K. Hale, Asymptotic Behavior of Dissipative Systems, AMS, Providence, RI, 1988.

[K] T. Kato, The Cauchy problem for quasi-linear symmetric hyperbolic systems, Arch. Rat. Mech. Anal. 58 (1975), 181-205.

[M] A. Mielke, The complex Ginzburg-Landau equation on large and unbounded domains: sharper bounds and attractors, Nonlinearity 10 (1997), 199-222.

[M-S] A. Mielke and G. Schneider, Attractors for modulation equations on unbounded domains-existence and comparison, Nonlinearity 8 (1995), 743-768.

[P] A. Pazy, Semigroups of Linear Operators and Applications to Partial Differential Equations, Springer, Berlin, 1983.

[T] R. Temam, Infinite-Dimensional Dynamical Systems in Mechanics and Physics, Springer, New York, 1997.

J. W. Cholewa and Tomasz Dlotko

Institute of Mathematics

Silesian University

Bankowa 14

40-007 Katowice, Poland

E-mail: jcholewa@ux2.math.us.edu.pl

tdlotko@ux2.math.us.edu.pl

Received March 11, 2004;

received in final form August 13, 2004 